\title{
IL PROFUMO. TRACCE EDUCATIVE NELLA MEMORIA E NELLA CULTURA DELLA SCUOLA,
}

por Miguel Beas Miranda. Ferrara, Edizioni Volta la carta, 2014, 130 páginas. ISBN: 978-88- 8908-370-98.

\section{SCUOLA DI ODORI, ODORI DI SCUOLA}

\section{Il senso identitario del profumo}

Un gioco di parole questo titolo, non vi è dubbio; ma al di là dell'immagine che tenta di «scherzare» con le parole, vi è il desiderio autentico di sintetizzare la forte e positiva impressione suscitata dal saggio di Beas Miranda Il profumo. Tracce educative nella memoria e nella cultura della scuola (Ferrara: Edizioni Volta la carta, 2014, Collana editoriale «Terre di Frontiera» diretta da Anita Gramigna) Si tratta di un lavoro significativo in quanto riesce a mettere insieme la vocazione didattica del docente con le aspirazioni, le curiosità e le esperienze degli studenti. Chi insegna si fa qui maestro di un'ermeneutica apparentemente semplice, in quanto dovrebbe limitarsi ad indagare sulla elemantarità dei dati sensibili ed, invece, finisce per aiutare chi apprende a riconoscere la complessità dei percorsi che si intrecciano tra sensibilità, sentimenti e ragione. Ecco perché si può parlare di una "scuola di odori»: si apprende a ricordare attraverso un senso, l'olfatto, che la tradizione culturale non ha tenuto in gran conto. Non è la vista, indispensabile per scrivere e leggere, benché le tecniche di pedagogia speciale abbiano insegnato ai non vedenti a sostituirla con la sensazione tattile; non è l'udito, indispensabile per dare il meglio nell'esperienza musicale, per quanto non siano mancati musicisti sordi o vicini alla sordità. $\mathrm{Al}$ massimo, l'olfatto viene associato al gusto: sensazioni che hanno a che fare con il mangiare ed il bere, attività cui ancora si fatica ad attribuire un autentico valore culturale. Da italiana, la speranza è che Expo di Milano 2015 possa contribuire, a livello mondiale, a lasciare un'impronta indelebile sulla cultura del cibo. 
Certo, gli studenti di Miguel Beas non si sarebbero aspettati un corso universitario storico-pedagogico con queste caratteristiche. Un approccio che affidava loro un ruolo di protagonisti ed un compito da svolgere in prima persona, prendendo se stessi come oggetto di studio in relazione a quell'esperienza esistenziale, la scuola, che finisce per essere dirompente ed indimenticabile per tutti, nel bene e nel male. Si scopre così, ad anni di distanza, che, spesso, ricordiamo gli insegnanti per gli aromi che li accompagnavano e le stessi discipline possono riportare alla mente tracce di profumi, non sempre gradevoli. L'autore ha avuto anche il merito di lasciare ampio spazio alle parole con le quali i suoi studenti descrivono la relazione olfatto-memoria, scoprendo con stupore come la sensazione apre spazi del tutto inaspettati sul passato, rievoca emozioni, lascia trasparire la dolcezza degli affetti familiari. Gli odori ci segnano, ci imprimono un'identità riconoscibile, tanto è vero che l'odore della povertà si fa emarginazione, terribile da scoprire sulla propria persona, ma che dovrebbe aiutarci a riflettere anche quando la sensazione olfattiva ne coglie traccia nell'altro. Così questa indagine sugli odori si fa pedagogico-sociale, presenta un orientamento formativo e possiede strategie epistemologiche che lo sorreggono. La tesi che si intende sostenere è che il metodo autobiografico, caratterizzante il progetto didattico di Beas, può rappresentare un chiaro esempio applicativo di una pedagogia socialmente orientata, metodo democratico di orientamento del soggetto che non discrimina in base all'appartenenza di genere, o sociale, o etnica, e nemmeno in base all'età. Si scopre, allora, come gli scritti autobiografici lascino intendere che una grande quantità di ragazzi e ragazze non accetta più di riconoscersi stabilmente in un gruppo e di dare continuità nel tempo ad un'esperienza che pure è considerata fondamentale. Vi è un chiaro fenomeno di provvisorietà, non implicante affatto superficialità, ma bisogno di cambiamento e curiosità, di continua riorganizzazione e riaggregazione spazio-temporale, di adesione critica ai processi della realtà complessa, sotto molteplici forme e con rapidi mutamenti di analisi dei fatti e di efficacia delle risposte. Cosa ha a che fare tutto ciò con le sensazioni olfattive? Per quanto possa sembrare strano, esse non solo ci danno identità, ma ci differenziano, connotando i nostri gusti e la nostra storia personale.

La componente giovanile si caratterizza per la varietà dei comportamenti, delle scelte, anche politiche, di forme culturali che le sono proprie e, in generale, tendono a differenziarsi da tutto ciò che è tipico del mondo 
adulto. La questione è poco indagata, ma è nostra convinzione che la presa di distanza abbia molto a che fare con un impiego mirato di profumi, o fetori, che vogliono marcare un confine. Siamo di fronte ad un segno della cultura giovanile che rappresenta pure una frontiera. Per questo il punto d'incontro tra politiche giovanili e culture giovanili è fragile, discontinuo, rarefatto, poiché le prime sono in larga misura pensate da menti adulte e le seconde diffidenti proprio di quell'adultità individuata nei suoi risvolti, veri o presunti, di «colonizzazione». La logica del rifiuto non è solo tipica dell'età, ma nasce anche dall'esperienza storica dei gruppi giovanili, che non hanno mancato di connotarsi più per forme di rottura e di presa di distanza che per consenso o volontà di dialogo con chi, inevitabilmente, veniva individuato come potere. E l'odore del potere è insopportabile, soprattutto se si vuole presentare con suadente, gradevole fragranza.

\section{Il suo significato culturale}

Bisogna avere il coraggio di continuare a credere nell'inesauribile radicalismo della narrazione popolare, ed il lavoro d'indagine di Beas si colloca su questa strada «dissidente», in certa misura anti-accademica proprio per il fatto che ha deciso di fare ricerca sul senso più umile, si potrebbe quasi dire "plebeo». In uno dei suoi racconti di Ragazzi di vita (1955), Pasolini accompagna il marginale Riccetto tra i banchi del mercato di un quartiere popolare di Roma. È l'odore del formaggio a far scattare in lui l'urgenza di dare risposta allo stimolo della fame e, quindi, a rubare un pezzo di cacio e a fuggire inseguito dal venditore; ma non sarà questo a placare l'eterna fame, che è pure pro il titolo dell'ultimo capitolo da cui è tratta la vicenda. Nelle narrazioni dei ragazzi spagnoli, per fortuna, quella fame inestinguibile non si avverte, sono altri tempi eppure si sentono richiami di sentori diversi, di campagne, di monti e di mari che si caratterizzano per difformità profonde.

Nel quadro dirompente di un mondo multietnico permane, ancora insostituibile, la narrazione plurima degli esseriumani, quali portatori di storie «locali» che non vogliono lasciarsi globalizzare e cadere nell'omologazione, quella degli odori rappresenterebbe forse la massima sconfitta del diritto alla differenza dissidente e consapevole. La possibilità che il «miracolo» della diversità narrativa sopravviva è forse legata al fatto di rappresentare una realtà connaturata al nostro essere, come sosteneva Barthes: 
Il racconto è presente in tutti i tempi, in tutti i luoghi, in tutte le società; il racconto comincia con la storia stessa dell'umanità; non esiste, non è mai esistito, in alcun luogo un popolo senza racconto. ${ }^{1}$

Occorre, perciò, la pazienza di ascoltare le storie degli altri per comprendere il presente, in quella dimensione di forte valenza formativa che chiamiamo «interculturalità». Essa è il segno evidente di un tempo e di uno spazio deve le culture s'intrecciano non tanto per scelta, quanto per condizioni di necessità percepite, di frequente, non in qualità di occasioni positive. Del resto la storia dei poveri è segnata da tradimenti ricorrenti, fiducie tradite, buona volontà umiliata, derisa, trucidata. Non è mai mancata, e non manca nemmeno oggi, la discriminazione legata non solo agli odori personali ma anche a quelli della cucina familiare che, ormai, in gran parte di Europa «profuma» molti quartieri periferici.

La narrazione "popolare» costruisce conoscenza e la pone in evidenza per gli altri quale modalità relazionale e, in certa misura, diviene un nuovo modo di proporre letteratura. In realtà essa fa molto di più, come nel caso del lavoro di Beas: richiede ad ognuno di farsi soggetto-oggetto d'indagine socio-letteraria attraverso il racconto autobiografico e la memoria di un passato narrativo, in cui tutti ci possiamo riconoscere come fruitori di situazioni incancellabili, che ci hanno affascinato e coinvolto nell'intimità affettiva:

A volte le storie sino lette da un libro, altre volte raccontate a memoria, altre ancora inventate [...]. Si tratta di un rito che rassicura tanto i piccoli quanto i grandi, un dono reciproco che stabilisce un contatto, rinsalda il legame. ${ }^{2}$

È quel dono a rendere la quotidianità non banale, ripetitiva o alienante; tanto è vero che ognuno di noi può riscontrare il peso che i racconti hanno assunto nella propria vita, quasi si trattasse di un vero e proprio itinerario formativo:

Il patrimonio di storie di cui la persona dispone quando è adulta non costituisce solo la fonte della sua stabilità o delle sue trasfor-

\footnotetext{
${ }^{1}$ R. Barthes, Introduzione all'analisi strutturale dei racconti, in L'analisi del racconto, AA. VV. (Milano: Bompiani, 1969), 7.

${ }^{2}$ P. Jerdlowski, Storie comuni. La narrazione nella vita quotidiana (Milano: Bruno Mondadori, 2000), 127.
} 
mazioni, ma diventa al tempo stesso uno strumento di lavoro, una bussola per mezzo della quale orientarsi. ${ }^{3}$

È significativo che vari studenti associno il profumo dei dolci, mentre si stanno cuocendo nella cucina della nonna, alla dimensione più generale e profonda dell'intimità familiare, con i suoi affetti indelebili. Se compio uno sforzo istantaneo per orientarmi su ciò che vorrei raccontare della mia vita olfattiva, nell'orizzonte nebuloso dell'infanzia, mi accorgo che alcune immagini sono connesse in profondità con la presenza della mia nonna materna. Ero una bambina difficile, proprio in relazione agli odori e ai sapori, odiavo il brodo di carne di qualsiasi tipo, perché emanava un odore nauseante. Eppure il brodo della nonna era diverso, il suo odore forte diventava una fragranza, soprattutto quando accompagnava quella straordinaria minestra della festa che erano i passatelli.

Nell'inevitabile preoccupazione che comporta ad ognuno il ri-orientarsi per affrontare le insidie del mondo globale, lo sguardo non può che volgersi alle nuove generazioni per come si sono espresse nelle loro narrazioni; ma non per prefigurarne, con rassegnazione, l'adattamento passivo al modello dominante, quanto piuttosto per tentare di valorizzare le forme di pensiero individuale, e le strategie di un pensiero socialmente coinvolgente e motivante, che possano concedere loro una effettiva dignità di vita e, quindi, di scelta. Accanto a quella che Morin definisce la via esteriore di un «apprendistato alla vita», nella quale la conoscenza dei media è basilare, risulta indispensabile mantenere salda la via interiore:

Si dovrebbero insegnare soprattutto gli errori o le deformazioni che si verificano anche nelle testimonianze più sincere o convinte; si dovrebbe studiare il modo in cui la mente occulta i fatti che disturbano la sua visione delle cose; si mostrerà inoltre come questa visione delle cose dipende non tanto dalle informazioni ricevute quanto dal modo in cui è strutturato il modo di pensare. ${ }^{4}$

L'autobiografia può essere uno strumento efficace per dare forma alla via interiore, perché implica fare i conti soprattutto con i propri limiti, le

\footnotetext{
3 Jerdlowski, Storie comuni, 133.

${ }^{4}$ E. Morin, La testa ben fatta. Riforma dell'insegnamento e riforma del pensiero (Milano: Raffaello Cortina Editore, 2000), 80.
} 
incertezze e gli inevitabili sbagli. Se il sociologo francese ritiene che ciò, attraverso l'esame di sé, l'auto-analisi e l'autocritica, possa ed debba essere già fonte di apprendimento nella scuola primaria, a noi sembra che il metodo autobiografico finisca per rappresentare un buon esempio di continuità formativa, sullo stesso versante, in età giovanile ed adulta. È decisivo mantenere viva la parola spontanea, anche quella apparentemente banale del linguaggio parlato, perché è segno di vita come pratica quotidiana e di un rapporto concreto con le cose, che facilmente si smarrisce nei linguaggi on line mancanti di sfumature affettive e di contaminazioni sentimentali. Nei testi dei ragazzi spagnoli che parlano delle loro famiglie e degli ambienti di vita traspare invece proprio la freschezza radicale di emozioni e affetti. La narrazione di sé può avere una funzione di condivisione e di confronto, soprattutto quando si traduce in un lavoro che è, ad un tempo, individuale e collettivo. Gli studenti dell'esperienza descritta hanno capito che la ricostruzione olfattiva della vicenda scolastica non era solo un modo per imparare a storicizzare la propria vita, ma rappresentava anche la composizione di un mosaico che finiva per tracciare un preciso disegno del rapporto con il mondo scolastico nelle diverse fasi che avevano connotato un'esperienza comune. La storia di vita produce effettiva autoeducazione quando sa abbandonare le certezze facili dei luoghi comuni, i percorsi lineari e scontati, per affrontare la dimensione dell'incertezza, della frantumazione, del disorientamento che accompagnano momenti di ogni esistenza, e accettare i segni perduranti di quell'immaturità che non rappresenta affatto un fattore degenerativo ma una risorsa. Gli scritti autobiografici di cui abbiamo avuto testimonianza, evidenziano che un numero considerevole di ragazze e ragazzi vive il disorientamento perdurante rispetto ai valori comuni. Mentre si coglie il pericolo che la stessa vita di gruppo, quello stare assieme per giorni e per anni negli stessi luoghi, possa condurre a chiusure claustrofobiche. Il bisogno di riorganizzare la propria vita con più libertà e consapevolezza, è segno di una dignità di ricerca che, tuttavia, non vuole mascherare ricorrenti paure di fallimento; perché è questo il sentimento che si può individuare dietro la precarietà attuale. Accanto alla provvisorietà è possibile cogliere anche l'affinità partecipativa: un modo di rendersi protagonisti attivi in conformità alle proprie scelte, a volte per omogeneità di generazione, di stato sociale, di cultura e perfino di etnia. La componente giovanile si caratterizza, anche qui come sempre, per la varietà dei comportamenti, per le scelte differenti nei fatti da analizzare, per i registri linguistici impiegati, che vanno dalla nuda cronaca a slanci commoventi, 
poetici. Più difficile stabilire se, nella ricostruzione olfattiva del mondo scolastico, possono avere un peso, anche solo di sfumatura, le appartenenze politiche o le forme culturali che i ragazzi avvertono come proprie. Del resto la politica, ancor più della cultura, è di difficile collocazione in un universo giovanile di per sé fragile, discontinuo, rarefatto. Essa è individuata, in larga misura, come prodotto «tossico» di menti adulte che si abbatte sulla scuola deprimendola; mentre l'altra è sì un bene di cui ci si vorrebbe appropriare, ma non sembra avere un legame di causa-effetto con la scuola, anzi librarsi in un empireo lontano dove l'olfatto non può raggiungerla.

\section{Le sue poetiche}

Presa, ormai, dal fascino dell'autoanalisi olfattiva, dopo aver proposto il ricordo degli odori del cibo nell'infanzia, mi accingo, per avvicinarmi ad una conclusione, a riportare una suggestione dell'adolescenza. Mi colpì nell'analisi della sinestèsi, presentatami dalla docente di scuola superiore, in quanto forma poetica che trasla da un senso all'altro la qualità della sensazione stessa, il classico riferimento ad un verso del Pascoli: «odore di fragole rosse». Mi resi subito conto di come l'immagine visiva rafforzasse la sensazione olfattiva; tuttavia, anni dopo, mi trovai a pensare anche l'esatto opposto grazie ad una esperienza estetica di grande rilievo, che mi ha messo sulla strada della passione autobiografica. L'elemento dirompente mi è stato offerto da un lavoro sulla senilità di notevole significato e, benché rappresentasse solo una finzione cinematografica, ha dato al mondo della cultura spunti rilevanti in chiave di interpretazione psico-sociale e, a me, pedagogico-sociale. Il modello di partenza è il celebre film di Bergman Il posto delle fragole (1957), la trasposizione psicologica del 1978 è il saggio: Riflessioni sul ciclo della vita del Dr. Borg 5 di Erikson. Borg dopo le varie vicissitudini di un viaggio in compagnia della nuora Marianne, ritrova la casa delle vacanze estive e, mentre si siede sull'erba a mangiare piccole fragole in un luogo a lui solo noto e caro fin da bambino, ricorda amici e parenti o, meglio, li «vede» con gli occhi della memoria, compresa la cugina Sara suo primo amore fanciullesco. Il regista non può che insistere sul dato visivo, poiché la macchina da presa è fatta per questo. Ma il vecchio attore, che interpreta il protagonista, gusta i frutti ad occhi chiusi offrendo l'immagine di chi sta

${ }^{5}$ E. Erikson, «Riflessioni sul ciclo della vita del Dr. Borg», in L'adulto, a cura di E. Erikson (Roma: Armando, 1981). 
ricordando i profumi di un luogo magico che, come capita nell'infanzia, si percepisce quale esclusivo, come un bosco o una giungla dove gli altri non sono ammessi se non con il nostro consenso. È con stupore misto a malinconia che ci ritroviamo, da adulti, a riconoscere com'era illusorio il nostro sogno: quell'angolo di giardino non misura che pochi metri, quel fosso non è una specie di fiume amazzonico, quel cespuglio di rovi non ha nulla a che fare con la selva caraibica. Si ha l'impressione che il vecchio medico abbia sempre vissuto un po' in disparte in quell'ambiente gioioso, come un "osservatore isolato» sia pure sensibile e attento. Attraverso i meccanismi della memoria del protagonista, Erikson osserva quanta reticenza vi sia per l'adulto nel ricordare la propria infanzia, quasi si celasse, in quel mondo, una minaccia tesa a "sconvolgere la convinzione da parte degli adulti di occupare nell'universo un posto sicuro e garantito con un punto di vista ben definito». ${ }^{6}$

Attraverso questo bel saggio di Miguel Beas, i suoi studenti avranno la possibilità di rendersi partecipi di un'ecologia del ricordo che ci piace segnalare attraverso un richiamo aristotelico. Il grande filosofo greco, che dava molta importanza ai cinque sensi, ne identificava anche un sesto, definito senso comune, poiché si trattava di mettere i sensi in una comune sinergia, al fine di cogliere le determinazioni che associano più sensi, quali unità, numero, grandezza, figura, movimento e quiete. Ma, soprattutto, il sesto senso è a fondamento della consapevolezza della sensazione, di quel «sentir di sentire» che nessun senso può possedere senza gli altri. Non è un caso che l'educazione sensoriale sia stato uno strumento strategico messo in atto più dall'educazione degli adulti che da quella scolastica tradizionale. Oggi è più facile riconoscere che la formazione autentica può essere solo quella per tutta la vita, la quale, nella sua pur recente storia, ha sempre messo al centro il problema della creatività come antidoto nei confronti del sapere sterile, utilitaristico, obbligatorio e lontano dalle motivazioni del soggetto. Richmond, ad esempio, ${ }^{7}$ metteva in guardia rispetto al tenace pregiudizio, ricorrente nella cultura occidentale, di ritenere le ragioni dei sensi e del cuore inferiori a quelle della mente. A causa del suo perdurare, le forze dell'immaginazione si sono via via ridotte, come appare evidente nella massificazione del tempo libero, che risulta sempre meno produttivo e sempre più banalizzato e

\footnotetext{
${ }^{6}$ Erikson, «Riflessioni sul ciclo della vita del Dr. Borg», 47.

${ }^{7}$ Cfr. K. W. Richmond, Il teorema dell'educazione continua (Roma: Armando, 1982).
} 
codificato in senso consumistico; per questo l'educazione che sa armarsi dell'ecologia del ricordo dovrà mettere al primo posto la creatività e farla «camminare» assieme alla ragione.

Già nel 1955, del resto, Fromm aveva considerato che la parte più importante dell'educazione non è quella scolastica, ma quella che consente all'uomo di sentirsi a proprio agio nel mondo, sia sul piano sensibile che intellettivo:

Se l'uomo comprende il mondo e in tal modo si unisce ad esso col pensiero, egli crea la filosofia, la teologia, il mito e la scienza. Se l'uomo esprime la sua comprensione del mondo con i sensi, egli crea l'arte e il rituale, crea il canto, la danza, il dramma, la pittura, la scultura. ${ }^{8}$

Per fare questo, bisogna saper amare tutte le strade che portano alla valorizzazione dei talenti, e non è da escludere, tra queste, quella che impara a fidarsi del proprio fiuto.

Anita Gramigna

Università di Ferrara (Italia)

anita.gramigna@unife.it

${ }^{8}$ E. Fromm, Psicanalisi della società contemporanea (Milano: Edizioni di Comunità, 1971), 333. 